\title{
ORIGINAL
}

\section{VARIABILIDAD EN LA PRESCRIPCIÓN FARMACÉUTICA DE ATENCIÓN PRIMARIA DE CASTILLA LA MANCHA DURANTE 2003}

\author{
Francisco López de Castro, M $^{a}$ José Montero Fernández, Natalia Valles Fernández, Olga Fernández \\ Rodríguez, Gemma Alejandre Lázaro y Joaquín Chacón Fuertes.
}

Gerencia de Atención Primaria de Toledo. Servicio de Salud de Castilla-La Mancha (SESCAM).

\section{RESUMEN}

Fundamento: Detectar la variabilidad en la práctica clínica es importante, ya que puede implicar infra o sobreutilización de recursos. El objetivo del estudio fue estudiar en Castilla la Mancha la variabilidad de la prescripción farmacéutica en Atención Primaria, en función de determinadas características sociosanitarias de cada zona de salud.

Métodos: Se revisó en 180 zonas de salud la prescripción en 2003 de varios grupos terapéuticos, recogiendo las siguientes variables: número de envases prescritos por 100 habitantes, porcentaje de mayores de 65 años en la zona, frecuentación (consultas/1.000 habitantes), médicos/1.000 habitantes y distancia al hospital de referencia.

Resultados: La mayor variabilidad en la prescripción se encontró en los fibratos (coeficiente de variación de 42,95\%) y antiH2 $(38,61 \%)$. El grupo con mejor correlación entre su tasa de prescripción y las variables analizadas fue el de los inhibidores de la enzima de conversión de la angiotensina ( $\rho$ de Spearman promedio 0,719 ); por contra, los antidepresivos tuvieron una correlación débil $(0,324)$. El porcentaje de mayores de 65 años y la frecuentación son las variables que más aparecen de forma significativa en los modelos de regresión múltiple construidos para cada grupo terapéutico. El mayor coeficiente de determinación se encontró en el modelo de los inhibidores de la enzima de conversión de la angiotensina $\left(R^{2}=0,761\right)$. El coeficiente de determinación promedio de todos los grupos, ponderado en base al volumen de prescripción, fue de 0,492 .

Conclusiones: Se observa gran variabilidad en la prescripción farmacéutica según las distintas zonas. En general, las variables analizadas explican en parte dicha variabilidad.

Palabras clave: Variaciones en la práctica clínica. Prescripción de medicamentos. Revisión de la Utilización de Drogas. Atención primaria de salud.

\section{ABSTRACT \\ Variability in Primary Care Drug Prescription in 2003 in Castile-la Mancha, Spain}

Background: Detecting variability in clinical practice is important, given that it may entail the underuse or overuse of resources. This study was aimed at studying the variability of drug prescription in Primary Care in Castile-La Mancha in terms of certain sociosanitary characteristics of each health district.

Methods: The prescribing of various therapeutic groups in 2003 was reviewed in 180 health districts, the following variables having been gathered: number of packages having been prescribed per 100 inhabitants, percentage of inhabitants over age 65 in the district, visit rate (visits/1,000 inhabitants), physicians/1,000 inhabitants and distance to the reference hospital.

Results: The greatest degree of drug-prescribing variability was found regarding fibrates $(42.95 \%$ variance factor) and $\mathrm{H} 2$-blockers $(38.61 \%)$. The group showing the closest correlation between its prescription rate and the variables analyzed was that of the angiotensin-converting enzyme inhibitors (mean Spearman $\rho$ : 0.719); antidepressants however showing a weak correlation (0.324).The percentage of inhabitants over age 65 and the visit rate are the variables arising more significantly in the multiple regression models constructed for each treatment group. The highest determination coefficient was found in the angiotensin enzyme-converting inhibitors $\left(\mathrm{R}^{2}=0.761\right)$. The mean determination coefficient for all of the groups, weighted on the basis of the prescribing volume, was 0.492 .

Conclusions: A high degree of drug-prescribing variability was found to exist according to the different districts. Generally speaking, the variables analyzed explain part of this variability.

Key words: Clinical Practice Variations. Prescriptions, Drug. Drug Utilization Review. Primary Health Care.

Correspondencia:

Francisco López de Castro

Unidad Docente de Medicina de Familia de Toledo

C/ Barcelona, 2

45005 Toledo.

Correo electrónico flopez@ sescam.org 


\section{INTRODUCCIÓN}

En el año 1938 Alison Glover publicó un estudio $^{1}$ en el que daba cuenta de la gran variabilidad existente en las tasas de incidencia de amigdalectomía en escolares entre los distintos condados de Inglaterra. A partir de ese momento se multiplicaron los estudios sobre la «variabilidad en la práctica clínica», comparando tasas de procedimientos médicos o quirúrgicos y de resultados obtenidos por dichos procedimientos en distintas áreas geográficas o centros sanitarios. Unos años más tarde Wennberg dio un nuevo impulso a este tipo de estudios, resaltando en su «hipótesis de la incertidumbre» cómo el estilo de práctica de los médicos es el primer factor causante de la variabilidad en situaciones en las que no existen criterios de actuación unánimes y $\operatorname{claros}^{2,3}$.

La variabilidad en la práctica clínica se entiende como las variaciones sistemáticas (no debidas al azar) en las tasas de incidencia (estandarizadas por edad y sexo) de un procedimiento clínico a un determinado nivel de agregación de la población ${ }^{4}$. El interés suscitado por la variabilidad se debe a la posibilidad de que tras ella se esconda un uso inadecuado de los recursos, bien por sobreutilización o por infrautilización, pero que en cualquier caso implicaría una mala calidad de la atención ${ }^{5}$.

La mayoría de los estudios realizados sobre variabilidad en nuestro país se refieren al ámbito hospitalario y de forma mayoritaria a procesos quirúrgicos ${ }^{6,7}$. Aunque no tan numerosos también existen algunos trabajos realizados en atención primaria de salud que exploran la variabilidad en el consumo de recursos ${ }^{8}$, en la realización de actividades preventivas $^{9}$, en la calidad de la atención prestada en algunos procesos crónicos ${ }^{10,11}$, en la frecuentación a consultas ${ }^{12} \mathrm{o}$ en la prescripción de medicamentos ${ }^{13,14}$.

La prescripción farmacéutica es uno de los aspectos de la práctica clínica que más interés despierta en Atención Primaria, por la importancia económica y sociosanitaria que tiene y por ser donde se realizan la mayoría de las prescripciones. La prescripción es un fenómeno complejo influenciado por variables sociodemográficas, de oferta y demanda sanitara ${ }^{15,16}$.

La Comunidad Autónoma de Castilla-La Mancha cuenta con una superficie de 79.463 $\mathrm{km}^{2}$ y una población de 1.815 .781 habitantes, según el padrón de 2003. El 21,4\% de la población es mayor de 65 años. Está dividida en 180 zonas de salud, agrupadas en 8 Áreas de Salud. La población media de las zonas básicas de salud es de 9.172,2 habitantes, con un rango entre 324 y 35.018 .

El objetivo de este estudio fue conocer la variabilidad en la prescripción de algunos grupos terapéuticos en Castilla la Mancha, y secundariamente analizar los posibles factores explicativos de dicha variabilidad relacionados con las características sociosanitarias de las zonas de salud.

\section{MATERIAL Y MÉTODOS}

Se trata de un estudio observacional descriptivo. Se analizó el número de envases prescritos de varios grupos terapéuticos, realizadas en las 180 zonas básicas de salud de Castilla La Mancha en el año 2003. Por su elevado volumen de prescripción y/o gasto generado, los grupos seleccionados fueron los siguientes: A02BA (Antihistamínicos H2 [AntiH2]), A02BC (Inhibidores de la bomba de protones [IBP]), C09A y C09B (Inhibidores de la enzima de conversión de la angiotensina [IECAs]), C09C y C09D (Antagonistas selectivos de la angiotensina II [ARA II]), C10AA (Estatinas), C10AB (Fibratos), J01M (Quinolonas) y N06 (Antidepresivos).

Se recogió el número de envases prescritos por cada 100 habitantes de cada uno de los grupos terapéuticos estudiados, en cada 
zona de salud. También se estudiaron aquellas variables explicativas disponibles a nivel de zona de salud, como variables demográficas la distancia al hospital de referencia desde la cabecera de la zona y el porcentaje de personas mayores de 65 años. Como variable de utilización de servicios la frecuentación $\left(\mathrm{n}^{\mathrm{o}}\right.$ de consultas por 1.000 habitantes en 2003). Como variable de la oferta el número de médicos por 1.000 habitantes. Los datos de prescripción se extrajeron del programa Digitalis y nos fueron facilitados por el área de Farmacia del Servicio de Salud de Castilla-La Mancha (SESCAM), mientras que los correspondientes a las demás variables estudiadas proceden del Sistema de Información en Atención Primaria de las distintas Gerencias de Atención Primaria de Castilla La Mancha.

El análisis estadístico se hizo con el programa SPSS 9.0. Para la descripción de los resultados se utilizaron la media y la desviación típica. Como indicadores de la variabilidad se emplearon la razón de variación (high-low ratio), que nos indica cuántas veces es más frecuente la prescripción en una zona respecto a otra, y el coeficiente de variación, medida fiable, válida y ampliamente utilizada en los estudios de variabili$\operatorname{dad}^{17,18}$.

Para valorar la capacidad explicativa de la variabilidad del conjunto de variables estudiadas se construyó para cada grupo terapéutico un modelo de regresión múltiple, siendo la variable dependiente el número de envases por cada 100 habitantes, y las independientes la distancia al hospital, el porcentaje de sujetos mayores de 65 años, la frecuentación y el número de médicos por 1.000 habitantes. Dada la finalidad de la regresión, más predictiva que estimativa, se optó por un modelo máximo que minimizara la probabilidad de infraajuste (Enter).

También se calcularon los coeficientes de correlación de la variable dependiente con las independientes, utilizando la $\rho$ de Spear- man, una vez comprobada la no-normalidad de las variables mediante el test de Kolmogorov-Smirnoff.

\section{RESULTADOS}

De los grupos estudiados, los mayores volúmenes de prescripción (tabla 1) en 2003 correspondieron a los IBP (1.249.629 envases), los IECAs (1.155.512) y los antidepresivos (1.127.304). La mayor variabilidad en la prescripción se encontró en la de fibratos (con un coeficiente de variación de 42,95\%), antiH2 $(38,61 \%)$, ARA II $(37,10 \%)$ e IECAs $(37,06 \%)$. Por el contrario, la menor variabilidad se observó en la prescripción de antidepresivos $(29,72 \%)$ e IBP $(29,33 \%)$. En los grupos terapéuticos de antiH2 y quinolonas se encontraron razones de variación por encima de 10 , lo que indica que la prescripción por 100 habitantes en algunas zonas de salud fue 10 veces mayor que en otras.

Respecto a la correlación entre el número de envases prescritos por 100 habitantes y las variables explicativas de la zona de salud, en la tabla 2 se pueden apreciar los coeficientes de correlación bivariados de la prescripción de cada grupo terapéutico con las variables estudiadas. Se han encontrado correlaciones fuertes en la prescripción de IECAs (con coeficientes entre $+0,848$ y $+0,415)$, IBP (entre $+0,767 \mathrm{y}+0,466)$ y quinolonas (entre $+0,727$ y $+0,578$ ). Por contra, en la prescripción de antidepresivos y fibratos se encontraron correlaciones entre $+0,426$ y $+0,129$.

La variable explicativa más correlacionada con la prescripción fue el porcentaje de personas mayores de 65 años (coeficientes entre $+0,848$ y $+0,421$ ), seguida del número médicos/1.000 habitantes (entre $+0,837 \mathrm{y}$ $+0,351$ ) y de la frecuentación (entre $+0,775$ $\mathrm{y}+0,367)$, mientras que la variable distancia al hospital se correlacionó débilmente (entre $+0,578$ y $+0,273$ ) con la mayoría de las prescripciones. 
Tabla 1

Datos de la prescripción de cada grupo terapéutico. Castilla-La Mancha, 2003

\begin{tabular}{|l|c|c|c|c|c|c|}
\hline \multirow{2}{*}{ Grupo terapéutico (código) } & \multirow{2}{*}{$\begin{array}{c}\text { Envases } \\
\text { prescritos en 2003 }\end{array}$} & \multicolumn{4}{|c|}{ Núm de envases por 100 habitantes (1) } \\
\cline { 3 - 7 } & 404.376 & 27,89 & 10,77 & $7,02-76,8$ & 10,94 & $38,61 \%$ \\
\hline AntiH2 (A02BA) & 1.249 .692 & 83,35 & 24,45 & $32,1-196,3$ & 6,11 & $29,33 \%$ \\
\hline IBP (A02BC) & 1.155 .512 & 82,49 & 30,57 & $22,9-205,9$ & 8,99 & $37,06 \%$ \\
\hline IECA (C09A+B) & 790.217 & 51,34 & 19,05 & $13,0-118,9$ & 9,14 & $37,10 \%$ \\
\hline ARA II (C09C+D) & 972.129 & 66,93 & 23,24 & $21,2-172,5$ & 8,13 & $34,72 \%$ \\
\hline Estatinas (C10AA) & 88.487 & 5,68 & 2,44 & $1,6-15,8$ & 9,87 & $42,95 \%$ \\
\hline Fibratos (C10AB) & 201.305 & 13,72 & 4,48 & $3,6-38,8$ & 10,77 & $32,65 \%$ \\
\hline Quinolonas (J01M) & 1.127 .304 & 69,33 & 20,60 & $29,3-141,4$ & 4,85 & $29,72 \%$ \\
\hline Antidepresivos (N06) & & & DE $\%$ & RV \\
\hline
\end{tabular}

IBP: inhibidores de la bomba de protones. IECA: Inhibidores de la enzima de conversión de la angiotensina.

ARA II: Antagonistas selectivos de la angiotensina II.

DE: desviación estándar. RV: razón de variación (valor máximo/valor mínimo). CV: coeficiente de variación (DE/media x 100).

(1) Distribución del número de envases prescritos por 100 habitantes en las 180 zonas de salud de Castilla La Mancha.

\section{Tabla 2}

Coeficientes de correlación ( $\rho$ de Spearman) entre la prescripción de cada grupo terapéutico y las variables sociosanitarias estudiadas en cada zona de salud

\begin{tabular}{|c|c|c|c|c|}
\hline $\begin{array}{l}\text { Grupo Terapéutico (código) } \\
\text { [No de envases prescritos } \\
\text { por cada } 100 \text { habitantes] }\end{array}$ & $\begin{array}{c}\text { Población mayor de } \\
65 \text { años }(\%)\end{array}$ & $\begin{array}{l}\text { Distancia al } \\
\text { hospital de } \\
\text { referencia }(\mathrm{km})\end{array}$ & $\begin{array}{l}\mathrm{N}^{0} \text { de médicos } \\
\text { por } 1000 \\
\text { habitantes }\end{array}$ & $\begin{array}{c}\text { Frecuentación } \\
\text { (consultas/hab/año) }\end{array}$ \\
\hline AntiH2 (A02BA) & 0,697 & 0,435 & 0,589 & 0,660 \\
\hline IBP (A02BC) & 0,767 & 0,466 & 0,729 & 0,646 \\
\hline $\operatorname{IECA}(\mathrm{C} 09 \mathrm{~A}+\mathrm{B})$ & 0,848 & 0,415 & 0,837 & 0,775 \\
\hline ARA II $(\mathrm{C} 09 \mathrm{C}+\mathrm{D})$ & 0,503 & 0,379 & 0,431 & 0,510 \\
\hline Estatinas (C10AA) & 0,663 & 0,316 & 0,668 & 0,627 \\
\hline Fibratos $(\mathrm{C} 10 \mathrm{AB})$ & 0,421 & 0,273 & 0,351 & 0,367 \\
\hline Quinolonas (J01M) & 0,727 & 0,578 & 0,651 & 0,684 \\
\hline Antidepresivos (N06) & 0,426 & $0,129(1)$ & 0,347 & 0,393 \\
\hline
\end{tabular}

IBP: inhibidores de la bomba de protones. IECA: Inhibidores de la enzima de conversión de la angiotensina.

ARA II: Antagonistas selectivos de la angiotensina II.

(1) Corresponde a una $\mathrm{p}=0,08$; el resto de coeficientes de correlación corresponden a $\mathrm{p}<0,01$. 
Tabla 3

Modelos de regresión múltiple (método «Enter») de la prescripción de los grupos terapéuticos estudiados

\begin{tabular}{|c|c|c|c|c|c|c|}
\hline & B & DE & Beta & $\mathbf{t}$ & $\mathbf{p}$ & $\mathbf{R}^{2}$ \\
\hline \multicolumn{7}{|c|}{ Variable dependiente: AntiH2 ( $\mathrm{n}^{\circ}$ envases $/ 100$ habitantes) } \\
\hline (Constante) & 4,157 & 2,100 & & 1,979 & 0,049 & \multirow{5}{*}{0,460} \\
\hline Porcentaje de mayores de 65 años & 0,418 & 0,105 & 0,412 & 3,979 & 0,000 & \\
\hline Distancia al hospital & $1,994 \mathrm{E}-02$ & 0,019 & 0,066 & 1,029 & 0,305 & \\
\hline $\mathrm{N}^{\circ}$ de facultativos por 1.000 hab. & $-2,261$ & 0,875 & $-0,215$ & $-2,583$ & 0,011 & \\
\hline Frecuentación & 1,476 & 0,296 & 0,426 & 4,979 & 0,000 & \\
\hline \multicolumn{7}{|c|}{ Variable dependiente: Inhibidores de la bomba de protones ( $n^{0}$ envases/100 habitantes) } \\
\hline (Constante) & 36,547 & 4,056 & & 9,011 & 0,000 & \multirow{5}{*}{0,609} \\
\hline Porcentaje de mayores de 65 años & 0,834 & 0,203 & 0,362 & 4,109 & 0,000 & \\
\hline Distancia al hospital & $3,189 \mathrm{E}-02$ & 0,037 & 0,047 & 0,852 & 0,395 & \\
\hline $\mathrm{N}^{\circ}$ de facultativos por $1.000 \mathrm{hab}$. & 6,953 & 1,690 & 0,291 & 4,113 & 0,000 & \\
\hline Frecuentación & 1,442 & 0,573 & 0,183 & 2,518 & 0,013 & \\
\hline \multicolumn{7}{|c|}{ Variable dependiente: Inhibidores de la enzima conversora de angiotensina ( ${ }^{0}$ envases/100 habitantes) } \\
\hline (Constante) & 13,563 & 3,968 & & 3,418 & 0,001 & \multirow{5}{*}{0,761} \\
\hline Porcentaje de mayores de 65 años & 1,391 & 0,199 & 0,483 & 7,006 & 0,000 & \\
\hline Distancia al hospital & $-4,580 \mathrm{E}-02$ & 0,037 & $-0,054$ & $-1,251$ & 0,213 & \\
\hline $\mathrm{N}^{\circ}$ de facultativos por $1.000 \mathrm{hab}$. & 7,685 & 1,654 & 0,258 & 4,647 & 0,000 & \\
\hline Frecuentación & 2,432 & 0,560 & 0,248 & 4,343 & 0,000 & \\
\hline \multicolumn{7}{|c|}{ Variable dependiente: Antagonistas selectivos de la angiotensina II ( ${ }^{\circ}$ envases/100 habitantes) } \\
\hline (Constante) & 18,422 & 4,264 & & 4,320 & 0,000 & \multirow{5}{*}{0,288} \\
\hline Porcentaje de mayores de 65 años & 0,269 & 0,213 & 0,150 & 1,262 & 0,209 & \\
\hline Distancia al hospital & $3,790 \mathrm{E}-02$ & 0,039 & 0,071 & 0,963 & 0,337 & \\
\hline $\mathrm{N}^{\circ}$ de facultativos por 1.000 hab. & $-1,976$ & 1,777 & $-0,106$ & $-1,112$ & 0,268 & \\
\hline Frecuentación & 2,746 & 0,602 & 0,449 & 4,562 & 0,000 & \\
\hline \multicolumn{7}{|c|}{ Variable dependiente: Estatinas ( $\mathrm{n}^{\circ}$ envases $/ 100$ habitantes) } \\
\hline (Constante) & 21,615 & 4,278 & & 5,053 & 0,000 & \multirow{5}{*}{0,519} \\
\hline Porcentaje de mayores de 65 años & 0,721 & 0,214 & 0,330 & 3,368 & 0,001 & \\
\hline Distancia al hospital & $-6,557 \mathrm{E}-02$ & 0,039 & $-0,101$ & $-1,661$ & 0,098 & \\
\hline $\mathrm{N}^{\circ}$ de facultativos por $1.000 \mathrm{hab}$. & 4,334 & 1,783 & 0,191 & 2,430 & 0,016 & \\
\hline Frecuentación & 2,424 & 0,604 & 0,324 & 4,014 & 0,000 & \\
\hline \multicolumn{7}{|c|}{ Variable dependiente: Fibratos ( $\mathrm{n}^{0}$ envases/100 habitantes) } \\
\hline (Constante) & 2,598 & 0,592 & & 4,392 & 0,000 & \multirow{5}{*}{0,165} \\
\hline Porcentaje de mayores de 65 años & $8,560 \mathrm{E}-02$ & 0,030 & 0,373 & 2,892 & 0,004 & \\
\hline Distancia al hospital & $3,110 \mathrm{E}-03$ & 0,005 & 0,046 & 0,570 & 0,570 & \\
\hline $\mathrm{N}^{\circ}$ de facultativos por $1.000 \mathrm{hab}$. & $-0,388$ & 0,247 & $-0,163$ & $-1,574$ & 0,117 & \\
\hline Frecuentación & 0,112 & 0,084 & 0,142 & 1,336 & 0,183 & \\
\hline \multicolumn{7}{|c|}{ Variable dependiente: Quinolonas ( $\mathrm{n}^{0}$ envases/100 habitantes) } \\
\hline (Constante) & 4,048 & 0,801 & & 5,052 & 0,000 & \multirow{5}{*}{0,547} \\
\hline Porcentaje de mayores de 65 años & 0,143 & 0,040 & 0,340 & 3,578 & 0,000 & \\
\hline Distancia al hospital & $4,125 \mathrm{E}-02$ & 0,007 & 0,329 & 5,580 & 0,000 & \\
\hline $\mathrm{N}^{\circ}$ de facultativos por 1.000 hab. & $-0,702$ & 0,334 & $-0,160$ & $-2,103$ & 0,037 & \\
\hline Frecuentación & 0,491 & 0,113 & 0,340 & 4,337 & 0,000 & \\
\hline \multicolumn{7}{|c|}{ Variable dependiente: Antidepresivos ( $\mathrm{n}^{0}$ envases/100 habitantes) } \\
\hline (Constante) & 3,948 & 0,478 & & 8,267 & 0,000 & \multirow{5}{*}{0,236} \\
\hline Porcentaje de mayores de 65 años & $6,249 \mathrm{E}-02$ & 0,024 & 0,322 & 2,615 & 0,010 & \\
\hline Distancia al hospital & $-7,964 \mathrm{E}-03$ & 0,004 & $-0,138$ & $-1,807$ & 0,072 & \\
\hline $\mathrm{N}^{\circ}$ de facultativos por 1.000 hab. & $-4,347 \mathrm{E}-02$ & 0,199 & 0,022 & $-0,218$ & 0,827 & \\
\hline Frecuentación & 0,177 & 0,067 & 0,267 & 2,618 & 0,010 & \\
\hline
\end{tabular}

En la tabla 3 se presentan los modelos de regresión múltiple para cada grupo terapéutico. Los modelos en los que las variables estudiadas (porcentaje de mayores de 65 años, frecuentación, distancia y facultativos por mil habitantes) tienen una mayor capaci- 
dad para explicar la variabilidad observada en la prescripción fueron los de IECAs (coeficiente de determinación $\mathrm{R}^{2}=0,761$ ), IBP $\left(\mathrm{R}^{2}=0,609\right)$ y quinolonas $\left(\mathrm{R}^{2}=0,547\right)$. El coeficiente de determinación promedio, ponderado según el volumen de prescripción de cada grupo terapéutico, fue de 0,492 .

Las variables que más frecuentemente aparecieron asociadas de forma significativa en estos modelos explicativos fueron el porcentaje de mayores de 65 años (con coeficientes beta que oscilan entre $+0,15 \mathrm{y}+0,48)$ y la frecuentación (beta entre $+0,14$ y $+0,45)$, estadísticamente significativas en casi todos los modelos.

\section{DISCUSIÓN}

La inclusión en el estudio de algunas zonas con poblaciones pequeñas $y$, en consecuencia, pocas prescripciones hace posible que las razones de variación puedan estar afectadas por el azar. Por ello, como se comentó en al apartado de material y métodos, hemos preferido utilizar el coeficiente de variación como índice de variabilidad.

La variabilidad en la prescripción ya ha sido constatada en otros trabajos que estudiaban la utilización de algunos grupos terapéuticos concretos, como ansiolíticos ${ }^{14}$, antibióticos ${ }^{19} \mathrm{o}$ inhibidores de la bomba de protones ${ }^{20}$. Nuestro estudio pretende dar una idea global de la variabilidad en la prescripción, no limitada a un solo grupo de fárma$\cos$. En este sentido hemos encontrado coeficientes de variación en torno al $35 \%$ en casi todos los grupos estudiados, lo que indica la existencia de una importante variabilidad en la prescripción, mayor a la observada en los estudios antes mencionados, si bien es cierto que en ocasiones el ámbito del estudio, el nivel de agrupación de datos (nacionales o regionales) o el indicador de variabilidad son diferentes y no permiten una comparación clara. En cualquier caso, coeficientes de variación superiores al 33\% empiezan a tra- ducir dispersiones grandes ${ }^{21}$, por lo que podemos calificar de importante la variabilidad observada.

La variabilidad es diferente de un grupo terapéutico a otro, probablemente porque sus indicaciones no sean igual de claras y el grado de incertidumbre sobre su uso difiera de unos a otros. Así, en estudios sobre el consumo de medicamentos en países de la Unión Europea se han observado grupos terapéuticos, como los antihipertensivos o las benzodiacepinas, con coeficientes de variación superiores al 90\%, mientras otros, como los antidiabéticos -con pautas de uso bien establecidas-, apenas alcanzan el $20 \%{ }^{22}$.

Respecto a las causas de la variabilidad observada, atendiendo al coeficiente de determinación promedio encontrado $(0,492)$, podemos decir que casi la mitad de la misma podría explicarse por las variables estudiadas, factores influyentes «por el lado de la demanda» (población) en su mayoría. En general, las variables más influyentes son el porcentaje de personas mayores de 65 años y la frecuentación, variables básicamente dependientes de la población ${ }^{23}$ y en las que resulta comprensible una mayor prescripción en las zonas más envejecidas y demandantes de atención. De hecho, la proporción de pensionistas es una de las variables que mejor predice el gasto farmacéuti$\operatorname{co}^{24}$ y que resulta siempre asociada con la prescripción de medicamentos ${ }^{25,26}$.

En cuanto a la parte de la variabilidad no explicada por estos factores, aunque nuestro estudio no puede concluir nada al respecto, lo más probable es que proceda de factores influyentes «por el lado de la oferta», sobre todo aquellos que dependen del proveedor directo, esto es, del médico prescriptor y su 'estilo de práctica' que a juicio de los exper$\operatorname{tos}^{27,28}$ es el determinante fundamental de la variabilidad en la práctica clínica cuando no existen evidencias claras sobre el resultado de las diferentes intervenciones, es decir, en 
situaciones de «incertidumbre» sobre la estrategia clínica a seguir.

En resumen, existe una parte de la variabilidad en la prescripción no explicada por los factores dependientes de la población. Esto nos debe plantear dudas sobre la calidad de la atención, tanto en términos de equidad (infrautilización en determinadas zonas de salud) como de eficiencia (sobreutilización inadecuada en otras $)^{27}$, por lo que la calidad de la prescripción en aquellos grupos donde mayor variabilidad existe debería ser analizada.

\section{AGRADECIMIENTOS}

Al área de Farmacia del Servicio de Salud de Castilla-La Mancha y a las Gerencias de Atención Primaria por facilitarnos los datos necesarios para realizar este trabajo. Al Dr. D. José Conde Olasagasti por sus acertados comentarios y sugerencias.

\section{BIBLIOGRAFÍA}

1. Glover JA. The incidence of tonsillectomy in school children. Proc R Soc Med 1938; 31:1219-36.

2. Wennberg JE, Gittelsohn AM. Variations in medical care among small areas. Sci Am 1982; 264:100-1.

3. Wennberg JE. Dealing with medical practice variations: a proposal for action. Health Aff 1984; 3:632.

4. McPherson K. Cómo debería modificarse la política sanitaria ante la evidencia de variaciones en la práctica médica. Var Pract Med 1995; 7:9-17.

5. Delgado R, Carrasco G. Variabilidad no deseable en la práctica clínica. Rev Calidad Asistencial 2000; 15:639-40.

6. Jané E, Barba G, Salvador X, Salas T, Sánchez E, Bustins M. Variaciones en la tasa de hospitalización por procedimientos quirúrgicos seleccionados. Aplicaciones del análisis de áreas pequeñas. Gac Sanit 1996; 10:211-9.

7. Marques JA, Peiró S, Medrano J, Librero J, Meneu R, López Reneo R. Variabilidad en la práctica clíni- ca. Disponibilidad de recursos y utilización de procedimientos quirúrgicos. Gest Hosp 2001; 12:196201.

8. Molinos Castro S, González Martínez R, Cimas JE, Cabal A y Carril E. Variabilidad de la práctica médica ante una sinusitis en función del consumo de recursos. Aten Primaria 2000; 26:203-9.

9. Ruiz Pérez I, Ramos Rincón JM, Hernández-Aguado I. Variaciones en la prevención del riesgo cardiovascular: estudio poblacional. Gac Sanit 2003; $17: 20-6$

10. Mirá JJ, Llinás G, Gil V, Lorenzo S, Palazón I y Orozco D. Variabilidad en la atención a pacientes diabéticos e hipertensos en función de los estilos de práctica del médico. Aten Primaria 1999; 23:73-81.

11 Saturno Hernández PJ, Gascón Cánovas JJ, Bueno JM, Alcaraz J y Martínez Martínez P. El diagnóstico de las dislipemias en atención primaria: un servicio a mejorar. Resultados de una evaluación multicéntrica. Aten Primaria 2000; 25:82-8.

12. Rodríguez Ortiz de Salazar B, Martín Galilea MJ. Variabilidad en la utilización de recursos en atención primaria. Aten Primaria 1999; 23:110-5.

13. Arnau de Bolós JM, Bordas Julve JM, Casajuana Brunet J, Diògene Fadini E, Hernández Huet E, Llop Rius R et al. Variabilidad de la prescripción en indicaciones prevalentes en atención primaria: un estudio multicéntrico. Aten Primaria 1998; 22:41723.

14. Vega Alonso AT, Larrañaga Padilla M, Zurriaga Lloréns O, Gil Costa M, Urtiaga M y Calabuig Pérez J. Trastornos de ansiedad. Variabilidad y condicionantes de la actitud terapéutica de los médicos de atención primaria. Aten Primaria 1999; 24:56978 .

15. Caamaño F, Figueiras A, Gestal-Otero JJ. Condicionantes de la prescripción en atención primaria. Aten Primaria 2001; 27:43-8.

16. Abanades Herranz JC, Cabedo García V, Cunillera Grañó R, García Díez JJ, Jolín Garijo L, Martín Bun $\mathrm{M}$ et al. Factores que influyen en la prescripción farmacológica del médico de atención primaria. Aten Primaria 1998; 22:391-8.

17. Oterino de la Fuente D y Peiró S. Evolución de la hospitalización infantil en España. Variabilidad entre comunidades autónomas. Gac Sanit 2001; 15(1): 14-7. 
18. Fiol M, Cabadés A, Sala J, Marrugat J, Elosua R, Vega $\mathrm{G}$ et al, en representación de los investigadores del estudio IBERICA. Variabilidad en el manejo hospitalario del infarto agudo de miocardio en España. Estudio IBERICA. Rev Esp Cardiol 2001; $54: 443-52$

19. Cars O, Mölstad S, Melander A. Variation in antibiotic use in the European Union. Lancet 2001; 357:1851-3.

20. Jones MI, Greenfield SM, Jowett S, Bradley CP, Seal R. Proton pump inhibitors: a study of GPs' prescribing. Fam Pract 2001; 18:333-8.

21. Carrasco de la Peña JL. El Método Estadístico en la

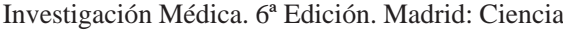
3; 1995.

22. González López-Valcárcel B, López Cabañas A, Cabeza Mora A, Díaz Berenguer JA, Ortún V, Alamo Santana F. Estudios de Utilización de Medicamentos y registros de datos en Atención Primaria (2005). Disponible en: http://www.econ.upf.es/docs/ papers/downloads/809.pdf [consultado el 21 de julio de 2005].

23. Meneu R. Variabilidad de las decisiones médicas y su repercusión sobre las poblaciones. Barcelona: Masson; 2002.
24. García-Sempere A y Peiró S. Gasto farmacéutico en atención primaria: variables asociadas y asignación de presupuestos de farmacia por zonas de salud. Gac Sanit 2001; 15:32-40.

25. Schwarz Chávarri H, Pedrera Carbonell V, Ortuño López JL, Orozco Beltrán D, Gil Guillén V, Pastor Climent MA. Influencia de las características de los médicos y del cupo en la vacunación antigripal de ancianos en un área de salud de la Comunidad Valenciana. Aten Primaria 2004; $34: 336-42$.

26. Catalán Ramos A, Madridejos Mora R, Font i Pous M, Pane Mena O, Jiménez Villa J, Huguet Recasens M. Factores asociados a la prescripción de medicamentos. Gac Sanit 1989; 3(14):497501.

27. Marión Buen J, Peiró S, Márquez Calderón S, Meneu de Guillerna R. Variaciones en la práctica médica: importancia, causas e implicaciones. Med Clin (Barc) 1998; 110:382-90.

28. Gómez de la Cámara. La medicina basada en evidencias científicas: mito o realidad de la variabilidad de la práctica clínica y su repercusión en los resultados de salud. An Sist Sanit Navar 2003; 26:11-26. 